\title{
Super stellar clusters with a bimodal hydrodynamic solution: an approximate analytic approach
}

\author{
R. Wünsch ${ }^{1}$, S. Silich ${ }^{2}$, J. Palouš ${ }^{1}$, and G. Tenorio-Tagle ${ }^{2}$ \\ 1 Astronomical Institute, Academy of Sciences of the Czech Republic, v.v.i., Boční II 1401, 14131 Prague, Czech Republic \\ e-mail: richard@wunsch.cz \\ 2 Instituto Nacional de Astrofísica Optica y Electrónica, AP 51, 72000 Puebla, Mexico
}

Received 12 February 2007 / Accepted 22 May 2007

\section{ABSTRACT}

\begin{abstract}
Aims. We look for a simple analytic model to distinguish between stellar clusters undergoing a bimodal hydrodynamic solution from those able to drive only a stationary wind. Clusters in the bimodal regime undergo strong radiative cooling within their densest inner regions, which results in the accumulation of the matter injected by supernovae and stellar winds and eventually in the formation of further stellar generations, while their outer regions sustain a stationary wind.

Methods. The analytic formulae are derived from the basic hydrodynamic equations. Our main assumption, that the density at the star cluster surface scales almost linearly with that at the stagnation radius, is based on results from semi-analytic and full numerical calculations.

Results. The analytic formulation allows for the determination of the threshold mechanical luminosity that separates clusters evolving in either of the two solutions. It is possible to fix the stagnation radius by simple analytic expressions and thus to determine the fractions of the deposited matter that clusters evolving in the bimodal regime blow out as a wind or recycle into further stellar generations.
\end{abstract}

Key words. galaxies: stellar clusters - ISM - kinematics and dynamics

\section{Introduction}

The discovery of young massive stellar clusters or super stellar clusters (hereafter SSCs) with typical masses between several times $10^{4} M_{\odot}$ to several times $10^{6} M_{\odot}$ and radii in the range 1-10 pc has unveiled the dominant mode of star formation in starburst and interacting galaxies (see, for example, McCrady et al. 2003; Whitmore 2006, and references therein). SSCs dominate the structure of the ISM in their host galaxies via their large UV photon output and their high velocity outflows or star cluster winds. Furthermore, the interaction of the winds from nearby SSC may lead to the inner structure of the large-scale galactic outflows, or super galactic winds, which connect starburst events with the intergalactic medium as in M 82 (Tenorio-Tagle et al. 2003).

Here, we center our attention on the hydrodynamics of the matter reinserted within the SSC volume, particularly in the case of massive and compact SSCs. The latter have been shown to be exposed to the strong radiative cooling (see Silich et al. 2004) that leads to a bimodal hydrodynamic solution (Tenorio-Tagle et al. 2007). This phenomenon occurs because the cluster mechanical luminosity and the injected gas density are nearly linear functions of the cluster mass, while the cooling rate is proportional to the square of the density, and therefore if one considers very massive and compact clusters, radiative cooling would always dominate (see Silich et al. 2003, for details). Radiative cooling depletes the energy in the densest inner regions enclosed within the so-called stagnation radius. The gas velocity at the stagnation radius is zero $\mathrm{km} \mathrm{s}^{-1}$. Thus the matter reinserted through stellar winds and supernovae within the central volume is accumulated to become thermally and/or gravitationally unstable leading to further episodes of star formation
(Tenorio-Tagle et al. 2005). On the other hand, the matter injected in the outer layers, between the stagnation radius and the cluster surface, composes a stationary wind. In the adiabatic solution and for low mass and extended clusters, the stagnation radius is always found at the star cluster center. However, if radiative cooling is taken into consideration, it progressively moves towards the star cluster surface as one considers more massive and compact clusters.

In this work, as in Chevalier \& Clegg (1985), the density, temperature and velocity are functions of the radial coordinate only. Such an approach does not take into account the effects of the turbulence generated by individual stellar winds and supernovae within the cluster volume. By changing the density, velocity and temperature distributions, it is thought to provide an additional transport of energy towards the smallest condensations. However it cannot prevent catastrophic cooling within the densest parts of the flow because it results from the net energy balance inside the cluster.

The properties of such clusters are described here by an approximate analytical formulation, in agreement with our former semi-analytic and numerical calculations (see Sects. 2 and 3). We display our results in a series of universal diagrams valid for all massive and compact SSCs. Section 4 deals with the impact of different heating efficiencies.

\section{Adiabatic vs. radiative solutions}

The original framework to study the fate of the matter deposited by supernovae and strong stellar winds within a star cluster volume was developed by Chevalier \& Clegg (1985). In their adiabatic model the kinetic energy released by individual sources is completely thermalized in situ via direct collisions of the high 
velocity gaseous streams from neighboring sources. This results in a high central temperature $\left(\sim 10^{7}-10^{8} \mathrm{~K}\right)$ and pressure that exceeds the pressure of the surrounding interstellar medium. The velocity of the injected matter then acquires a particular distribution, increasing from zero $\mathrm{km} \mathrm{s}^{-1}$ at the star cluster center to the speed of sound $\left(c_{\mathrm{SC}}\right)$ at the star cluster surface, approaching rapidly a supersonic value $\left(V_{\mathrm{A} \infty}\right)$ as it moves outside the cluster volume. $c_{\mathrm{SC}}$ is defined as: $c_{\mathrm{SC}}=[(\gamma-1) /(\gamma+1)]^{1 / 2} V_{\mathrm{A} \infty}$; where $V_{\mathrm{A} \infty}=\left[2 L_{\mathrm{SC}} / \dot{M}_{\mathrm{SC}}\right]^{1 / 2}$ is the adiabatic wind terminal velocity, $L_{\mathrm{SC}}$ is the star cluster mechanical luminosity, and $\dot{M}_{\mathrm{SC}}$ is the rate of mass injection provided by supernovae and stellar winds. The condition for a stationary flow is then:

$\dot{M}_{\mathrm{SC}}=4 \pi R_{\mathrm{SC}}^{2} \rho_{\mathrm{SC}} c_{\mathrm{SC}}$,

where $R_{\mathrm{SC}}$ is the radius of the cluster and $\rho_{\mathrm{SC}}$ is the density of the outflow at the star cluster surface. The relation holds whenever $\rho_{\mathrm{SC}}$ reaches the proper value, which is in direct proportion to the density at the stagnation radius (i.e. in the center), $\rho_{\mathrm{st}}: \rho_{\mathrm{SC}}=$ $[(\gamma+1) /(6 \gamma+2)]^{(3 \gamma+1) /(5 \gamma+1)} \rho_{\text {st }}$, where $\rho_{\text {st }}$ is (Cantó et al. 2000)

$\rho_{\mathrm{st}}=\left(\frac{\gamma+1}{\gamma-1}\right)^{1 / 2}\left(\frac{6 \gamma+2}{\gamma+1}\right)^{\frac{3 \gamma+1}{5 \gamma+1}} \frac{q_{m} R_{\mathrm{SC}}}{3 V_{\mathrm{A} \infty}}$.

Here $q_{m}=\left(3 \dot{M}_{\mathrm{SC}}\right) /\left(4 \pi R_{\mathrm{SC}}^{3}\right)$ is the mass deposition rate per unit volume which for a given $R_{\mathrm{SC}}$ scales linearly with the star cluster mass, $M_{\mathrm{SC}}$, and $\gamma$ is the ratio of specific heats. Note that in the adiabatic solution, if one fixes the radius of the cluster, $R_{\mathrm{SC}}$, and the adiabatic terminal velocity, $V_{\mathrm{A} \infty}$, the central density and the density at the star cluster surface grow linearly with the mass of the considered cluster, since the stagnation radius is located at the star cluster center and thus the density profiles remain self-similar.

It has recently been shown, however, that the adiabatic model is inadequate for very massive and compact star clusters, as in these cases radiative cooling becomes a dominant factor (Silich et al. 2004). The authors demonstrated that in the radiative case the wind density at the stagnation radius changes to

$\rho_{\mathrm{st}}=\mu_{i} q_{m}^{1 / 2}\left[\frac{\eta V_{\mathrm{A} \infty}^{2} / 2-c_{\mathrm{st}}^{2} /(\gamma-1)}{\Lambda\left(T_{\mathrm{st}}, Z\right)}\right]^{1 / 2}$,

where $\mu_{i}=14 / 11 m_{\mathrm{H}}$ is the mean mass per ion $\left(m_{\mathrm{H}}\right.$ is the proton mass), $\Lambda\left(T_{\mathrm{st}}, Z\right)$ is the cooling function (the cooling rate is $Q=$ $\left.n_{e} n_{i} \Lambda\left(T_{\mathrm{st}}, Z\right)\right), T_{\mathrm{st}}$ and $c_{\mathrm{st}}$ are the temperature and speed of sound at the stagnation radius, and $Z$ is the metallicity of the plasma. The parameter $\eta$ describes how much of the deposited energy is transformed into energy of the outflow (see Sect. 4 for a detailed discussion). We assume $\eta=1$ everywhere in Sects. 2 and 3.

For clusters of a given size, the density at the stagnation radius grows in all cases with the mass of the cluster. In the case of low mass clusters it results from a combination of two effects: i) the mass deposition rate per unit volume, $q_{m}$, is a linear function of $\dot{M}_{\mathrm{SC}}\left(\sim M_{\mathrm{SC}}\right)$; ii) the temperature at the stagnation radius, $T_{\text {st }}$, decreases with increasing cluster mass $M_{\mathrm{SC}}$ making $c_{\text {st }}$ smaller and thus the term in brackets in the rhs of Eq. (3) larger. The combined action of these two factors then causes that the radiative solution proposed by Silich et al. (2004) is almost identical to the adiabatic solution of Chevalier \& Clegg (1985).

However, if one considers more massive clusters (or clusters with a higher mechanical luminosity, $L_{\mathrm{SC}}$ ) one would soon reach a critical value for which strong radiative cooling would set in at the star cluster center, drastically changing the inner wind structure (see Tenorio-Tagle et al. 2007). For clusters above this limit, the temperature $T_{\mathrm{st}}$ at the stagnation radius $R_{\mathrm{st}}$ does not change any more and remains equal to the value that corresponds to the highest possible pressure there (see Sect. 3.1 below). Thus the second term in Eq. (3) remains fixed and the density at the stagnation radius, $\rho_{\mathrm{st}}$, grows as the square root of the star cluster mass, whereas the mass deposition rate continues to grow linearly with the mass of the considered cluster. This implies that above the threshold line, the stationary condition Eq. (1) cannot be fulfilled unless the stagnation radius moves towards the star cluster surface. Tenorio-Tagle et al. (2007) showed how to extend the semi-analytic approach proposed in Silich et al. (2004) for this case. The authors also found the semi-analytic solution for the outflow beyond the stagnation radius, and compared it with full numerical calculations performed with the finite difference hydrodynamic code ZEUS for which the cooling routine was modified to make it suitable for the modeling of extremely fast cooling regions. In particular, they include the cooling rate in the computation of the time-step assuming that the amount of energy that can be radiated away from a given cell during one timestep must be smaller than $10 \%$ of its internal energy. The timestep is decreased to meet this cooling rate condition. However, since this could lead to extremely small time-steps, which would substantially degrade the overall code performance, they do not allow the global time-step to decrease below 0.1 times the "hydrodynamic" time-step determined by the Courant-FriedrichLevi criterion. If a certain cell requires an even smaller time-step due to the cooling rate condition, the time-step was subdivided even further and then was used to numerically integrate the energy equation only in the affected cell(s). This "time refinement" was applied only locally, so the CPU time is not wasted in cells where the high time resolution is not required (see Tenorio-Tagle et al. 2007 for more details).

\section{The catastrophic cooling regime}

\subsection{The temperature at the stagnation radius}

Equation (3) allows one to determine the pressure at the stagnation radius $P_{\mathrm{st}}=\rho_{\mathrm{st}} k T_{\mathrm{st}} / \mu$ where $k$ is the Boltzmann constant, and $\mu=14 / 23 m_{\mathrm{H}}$ is the mean mass per particle. Tenorio-Tagle et al. (2007) showed that in the bimodal regime the pressure at the stagnation radius always attains the maximum value allowed by the shape of the cooling function, and thus one can find the corresponding temperature numerically if $V_{\mathrm{A} \infty}$ and the shape of the cooling function, $\Lambda(T, Z)$, are known.

Figure 1 displays $T_{\text {st }}$ for different values of the adiabatic wind terminal speed (or different ratios of energy to mass deposition rates) and different metallicities of the thermalized plasma. The temperature at the stagnation radius grows approximately as a quadratic function of $V_{\mathrm{A} \infty}$ and remains always below the adiabatic value (dotted line in Fig. 1). For practical purposes one can suggest that it does not depend on the plasma abundance (see Fig. 1). Note that for these calculations and throughout the paper we have used the Raymond et al. (1976) equilibrium cooling function tabulated and updated by Plewa (1995).

\subsection{The threshold mechanical luminosity}

In the case of a homogeneous stellar mass distribution, the equation of mass conservation

$\frac{1}{r^{2}} \frac{\mathrm{d}}{\mathrm{d} r}\left(\rho_{w} u_{w} r^{2}\right)=q_{m}$ 


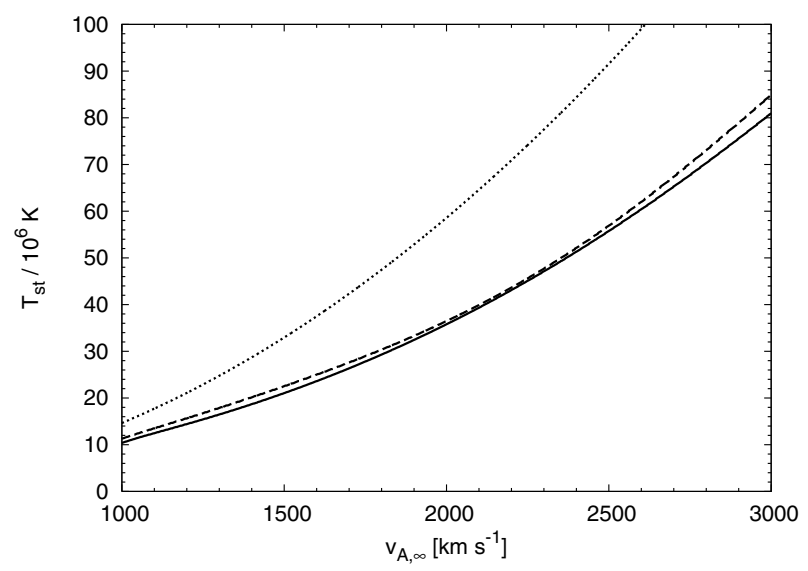

Fig. 1. The temperature at the stagnation radius as a function of the adiabatic wind terminal speed, $V_{\mathrm{A} \infty}=2 L_{\mathrm{SC}} / \dot{M}_{\mathrm{SC}}$. Solid and dashed lines represent the temperature at the stagnation radius for solar and 10 times solar metallicities, respectively. The adiabatic wind stagnation (central) temperature is shown by the dotted line.

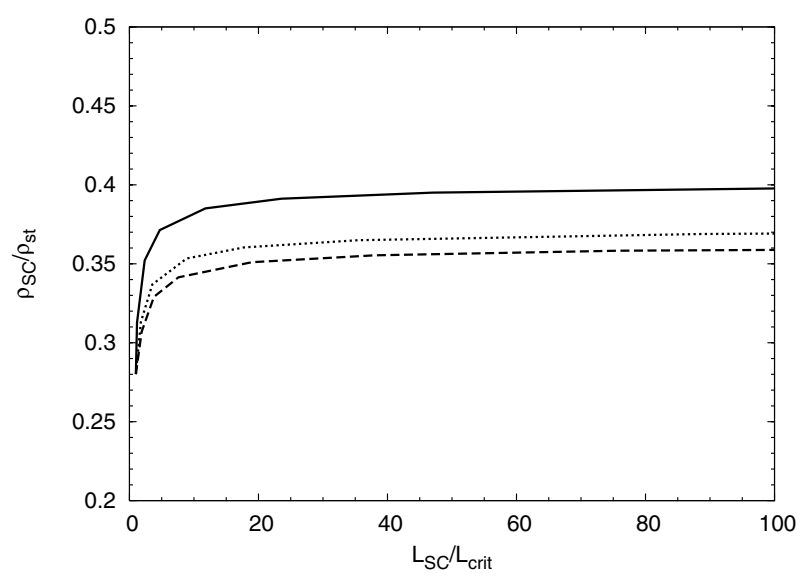

Fig. 2. The ratio of the density at the star cluster surface to that at the stagnation radius in the catastrophic cooling regime. Solid, dotted and dashed lines present semi-analytic results (see Silich et al. 2004) for $V_{\mathrm{A} \infty}=1000 \mathrm{~km} \mathrm{~s}^{-1}, V_{\mathrm{A} \infty}=1500 \mathrm{~km} \mathrm{~s}^{-1}$ and $V_{\mathrm{A} \infty}=2000 \mathrm{~km} \mathrm{~s}^{-1}$, respectively.

can be easily integrated. At the star cluster edge this results in:

$\rho_{\mathrm{SC}}=\frac{q_{m} R_{\mathrm{SC}}}{3 c_{\mathrm{SC}}}\left(1-\frac{R_{\mathrm{st}}^{3}}{R_{\mathrm{SC}}^{3}}\right)$.

As mentioned above, in the adiabatic case the density at the star cluster surface scales linearly with that at the central stagnation radius. This is not exactly the case in the radiative solution. Nevertheless, $\rho_{\mathrm{SC}} / \rho_{\mathrm{st}}$ remains restricted into a very narrow interval, $0.28 \leq \rho_{\mathrm{SC}} / \rho_{\mathrm{st}} \leq 0.42$, (see Fig. 2 ), which allows one to propose a simple analytic expression for the stagnation radius in reasonable agreement with the results found in semi-analytic and full numerical calculations. Indeed, assuming by analogy with the adiabatic solution, that $\rho_{\mathrm{SC}}=\alpha \rho_{\mathrm{st}}$, where $\alpha$ is a fiducial coefficient, one can combine Eqs. (5) and (3) to derive:

$\frac{R_{\mathrm{st}}^{3}}{R_{\mathrm{SC}}^{3}}=1-\left(\frac{L_{\mathrm{crit}}}{L_{\mathrm{SC}}}\right)^{1 / 2}$,

where $L_{\text {crit }}$ is

$L_{\mathrm{crit}}=\frac{6(\gamma-1) \pi \eta \alpha^{2} \mu_{i}^{2} R_{\mathrm{SC}} V_{\mathrm{A} \infty}^{4}}{(\gamma+1) \Lambda_{\mathrm{st}}}\left(\frac{\eta V_{\mathrm{A} \infty}^{2}}{2}-\frac{c_{\mathrm{st}}^{2}}{\gamma-1}\right)$.
In Eqs. (6) and (7) $\Lambda_{\text {st }}$ and $c_{\text {st }}$ are the cooling function value and speed of sound at the stagnation radius, respectively. To derive these equations we have used the adiabatic relation for the sound speed at the star cluster surface: $c_{\mathrm{SC}}^{2}=(\gamma-1) V_{\mathrm{A} \infty}^{2} /(\gamma+1)$. When the mechanical luminosity of the cluster exceeds $L_{\text {crit }}$, the stagnation radius detaches from the star cluster center, which implies that $L_{\text {crit }}$ is the threshold luminosity which separates clusters evolving in the catastrophic cooling (bimodal) regime from those evolving either in the quasi-adiabatic or in the radiative regimes, with their stagnation radius located at the star cluster center. Note that for the latter cases Eq. (6) is not valid.

\subsection{The analytic formulation}

The analytic values for the threshold luminosity and the stagnation radius have been compared with our semi-analytic results and full numerical calculations (see Figs. 2 and 3 in Tenorio-Tagle et al. 2007). For that we have used first Fig. 1 to obtain the temperature at the stagnation radius for a given value of the adiabatic wind terminal speed, $V_{\mathrm{A} \infty}$. We then use this temperature to calculate the sound speed, $c_{\mathrm{st}}$, and the corresponding cooling function value, $\Lambda_{\mathrm{st}}$, and then use these values to calculate the threshold luminosity and the stagnation radius from Eqs. (7) and (6). The agreement between the approximate analytic expressions and semi-analytic results is good if the fiducial coefficient $\alpha=0.28$ (see Fig. 3).

Using Eq. (6) one can also estimate the fraction of the deposited matter that clusters evolving in the bimodal regime return to the ISM of their host galaxy:

$\frac{\dot{M}_{\mathrm{out}}}{\dot{M}_{\mathrm{SC}}}=\frac{R_{\mathrm{SC}}^{3}-R_{\mathrm{st}}^{3}}{R_{\mathrm{SC}}^{3}}=\left(\frac{L_{\mathrm{crit}}}{L_{\mathrm{SC}}}\right)^{1 / 2}$.

This quantity decreases monotonically with the star cluster mechanical luminosity (see Fig. 3) as was also found in the $1 \mathrm{D}$ hydrodynamic simulations performed by Tenorio-Tagle et al. (2007).

The analytic expression (8) reproduces the numerical results with an accuracy better than $\sim 25 \%$. Minor differences between the analytic and the numerical outputs indicate that $\rho_{\mathrm{SC}} / \rho_{\mathrm{st}}$ deviates from the assumed constant value.

\section{Heating efficiency}

Here we follow the Chevalier \& Clegg (1985) formulation supported by full numerical calculations (see, for example Cantó et al. 2000; Rockefeller et al. 2004) and assume that the injected matter is thermalized in situ via random encounters of high velocity stellar winds and supernovae ejecta. The efficiency of this process is an important, but poorly known parameter (Stevens \& Hartwell 2003). This parameter has been discussed by different groups (see, for example, Bradamante et al. 1998; Melioli $\&$ de Gouveia Dal Pino 2004, and references therein) who argued for very different values of heating efficiency that vary from $100 \%$ to a few per cent.

Note that different authors have different prescriptions for the physical processes that may lead to an incomplete transformation of the deposited kinetic energy. In our view, the high density of the sources may cause an important fraction of the star cluster mechanical luminosity to be immediately radiated away. This would happen locally at the sites where the ejecta from nearby stellar winds and supernovae would interact, inhibiting in this way the possibility of having such energy evenly 

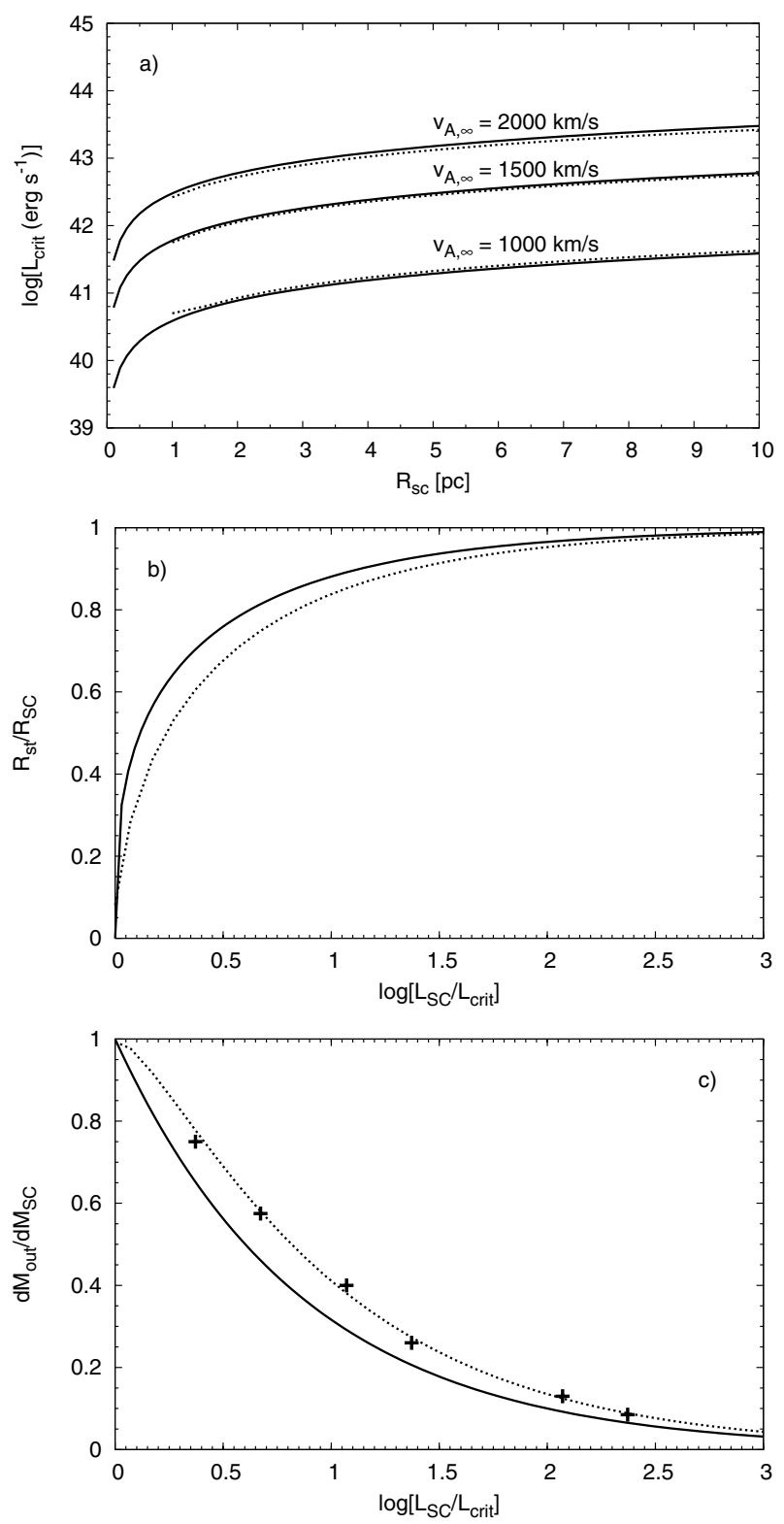

Fig. 3. The comparison of analytic results with semi-analytic and full numerical calculations. Panel a) displays the critical luminosity calculated for three different values of the adiabatic wind terminal speed: $V_{\mathrm{A} \infty}=1000 \mathrm{~km} \mathrm{~s}^{-1}, V_{\mathrm{A} \infty}=1500 \mathrm{~km} \mathrm{~s}^{-1}$ and $V_{\mathrm{A} \infty}=2000 \mathrm{~km} \mathrm{~s}^{-1}$. Panel b) displays how the stagnation radius shifts from the center when the mechanical luminosity exceeds the critical value. Panel c) shows the fraction of the matter supplied by supernovae and stellar winds that the cluster returns to the ISM of the host galaxy. In all panels solid lines present the analytic results. The results from the semi-analytic and full numerical calculations are shown by the dotted line and by the cross symbols, respectively. The fiducial coefficient $\alpha$ was set to $\alpha=0.28$ in all calculations.

spread throughout the cluster volume. Turbulence may also absorb some fraction of the deposited energy. The immediate consequence of such a sudden depletion of energy is to reduce the thermal pressure gradient responsible for the acceleration of the injected matter and thus the magnitude of the resultant high velocity outflow. We have incorporated all uncertainties related to this process into a parameter $\eta$ that represents the fraction of the deposited mechanical energy per unit time which is finally evenly spread throughout the cluster volume and participates in the thermal acceleration of the injected matter. The heating
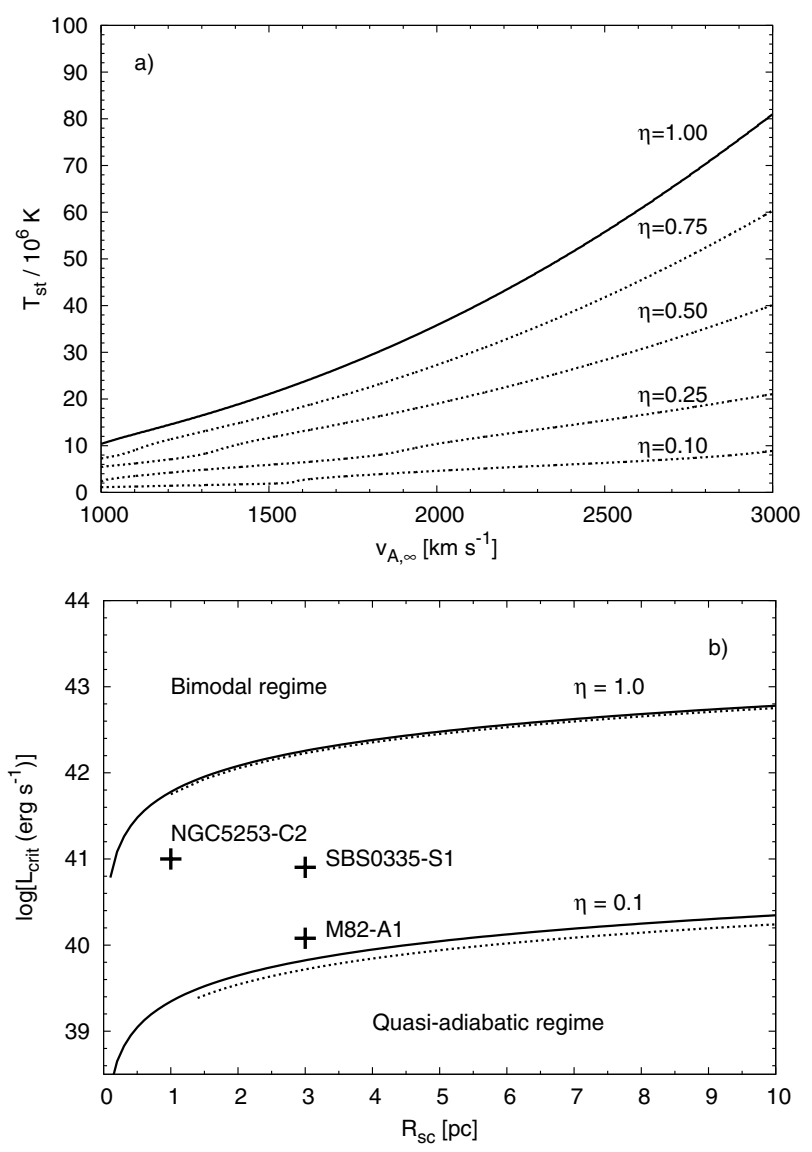

Fig. 4. The impact of the heating efficiency. Panel a) displays the temperature at the stagnation radius for different heating efficiencies, $\eta$. Panel b) displays the threshold mechanical luminosity for two heating efficiencies, $\eta=1$ and $\eta=0.1$. The analytic results (solid lines) are compared with the semi-analytic calculations (see Silich et al. 2006) indicated by dotted lines. The location of several massive SSCs with respect to the threshold lines are indicated in panel $\mathbf{b}$ ) by cross symbols (see Turner et al. 2003; Turner \& Beck 2004; Thompson et al. 2006; Smith et al. 2006).

efficiency then effectively reduces the value of the deposited energy, $L_{\mathrm{SC}}$, but does not affect the mass deposition rate, $\dot{M}_{\mathrm{SC}}$.

Figure 4 shows the impact of a reduced heating efficiency on the plasma temperature and how it drastically decreases the threshold luminosity. What the most reasonable value of the heating efficiency in super star clusters is, remains an open question in need of both detailed full hydrodynamical calculations and careful analysis of the available observational data. Nevertheless, the threshold mechanical luminosities calculated for a reasonable range of heating efficiencies, $0.1<\eta<1$ (shown in Fig. 4b), suggest that many SSCs may be found well above the threshold line, as in the case of the M 82-A1 supercluster. See Smith et al. (2006), who, based on the size of its associated HII region, estimated a heating efficiency $\eta<0.1$. A critical revision of the available super star cluster data will be the subject of a forthcoming communication.

\section{Conclusions}

We have provided a simple analytic formulation that allows for an easy determination in the energy deposition rate vs cluster size diagram, of the location of the threshold mechanical luminosity that separates clusters evolving in either of the two 
possible hydrodynamic solutions: the normally assumed negative feedback solution in which all mass supplied by $\mathrm{SNe}$ and individual stellar winds leaves the cluster as a cluster wind $\left(R_{\mathrm{st}}=0\right)$ and the bimodal solution $\left(0<R_{\mathrm{st}}<R_{\mathrm{SC}}\right)$ in which the reinserted matter accumulates within the volume defined by $R_{\text {st }}$. Simple analytic expressions, accounting also for a reduced heating efficiency, allow one to calculate the position of the stagnation radius and the fraction of the deposited matter that clusters evolving in the bimodal regime return to the ISM of their host galaxy. Our results are in good agreement with our former semi-analytic and numerical results.

Acknowledgements. We thank our anonymous referee for multiple comments and suggestions that greatly improved the paper. This study was partly supported by the Institutional Research Plan AV10030501 of the Astronomical Institute, Academy of Sciences of the Czech Republic and project LC06014 Center for Theoretical Astrophysics. We also acknowledge support from Conacyt (México) grant 47534-F and grants AYA2004-08260-C03-01 and AYA 2004-02703 from the Spanish Ministerio de Educación y Ciencia.

\section{References}

Bradamante, F., Matteucci, F., \& D'Ercole, A. 1998, A\&A, 337, 338 Cantó, J., Raga, A. C., \& Rodríguez, L. F. 2000, ApJ, 536, 896

Chevalier, R. A., \& Clegg, A. W. 1985, Nature, 317, 44

McCrady, N., Gilbert, A. M., \& Graham, J. R. 2003, ApJ, 596, 240

Melioli, C., \& de Gouveia Dal Pino, E. M. 2004, A\&A, 424, 817

Plewa, T. 1995, MNRAS, 275, 143

Raymond, J. C., Cox, D. P., \& Smith, B. W. 1976, ApJ, 204, 290

Rockefeller, G., Fryer, C. L., Melia, F., \& Warren, M. S. 2004, ApJ, 604, 662

Silich, S., Tenorio-Tagle, G., \& Muñoz-Tuñón, C. 2003, ApJ, 590, 791

Silich, S., Tenorio-Tagle, G., \& Rodríguez-González, A. 2004, ApJ, 610, 226

Silich, S., Tenorio-Tagle, G., \& Muñoz-Tuñon, C. 2006, in Triggered Star Formation in a Turbulent ISM, IAU Symp., 237

Smith, L. J., Westmoquette, M. S., Gallagher, J. S., et al. 2006, MNRAS, 370, 513

Stevens, I. R., \& Hartwell, J. M. 2003, MNRAS, 339, 280

Tenorio-Tagle, G., Silich, S., \& Muñoz-Tuñón, C. 2003, ApJ, 597, 279

Tenorio-Tagle, G., Silich, S., Rodríguez-González, A., \& Muñoz-Tuñón, C. 2005, ApJ, 628, L13

Tenorio-Tagle, G., Wünsch, R., Silich, S., \& Palouš, J. 2007, ApJ, 658, 1196

Thompson, R. I., Sauvage, M., Kennicutt, Jr., R. C., Engelbracht, C. W., \& Vanzi, L. 2006, ApJ, 638, 176

Turner, J. L., \& Beck, S. C. 2004, ApJ Let, 602, L85

Turner, J. L., Beck, S. C., Crosthwaite, L. P., et al. 2003, Nature, 423, 621

Whitmore, B. C. 2006 [arXiv: astro-ph/0612695] 